Research Paper

\title{
Knockdown of Yes-Associated Protein Induces the Apoptosis While Inhibits the Proliferation of Human Periodontal Ligament Stem Cells through Crosstalk between Erk and Bcl-2 Signaling Pathways
}

\author{
Yong Wen ${ }^{1,2}$, Yawen Ji1,2, Yunpeng Zhang1,2, Baoqi Jiang1,2, Cuizhu Tang1,2, Qi Wang1,2, Xiyan Chen², \\ Linglu Jia1, ${ }^{1,}$ Weiting $\mathrm{Gu}^{3 凶}$, Xin $\mathrm{Xu}^{1,2}$ \\ 1. School of Stomatology, Shandong University, Jinan, China; \\ 2. Shandong provincial key laboratory of oral tissue regeneration, Jinan, China; \\ 3. Qilu hospital of Shandong University, Jinan, China. \\ $\triangle$ Corresponding authors: Weiting Gu (weitinggu@gmail.com) No. 107, Wenhua Xi Road, Jinan, Shandong, 250012 P.R. China Tel./Fax: +86-531-82169268 Xin \\ Xu (xinxu@sdu.edu.cn) No. 44-1, Wenhua Xi Road, Jinan, Shandong, 250012 P.R. China Tel./Fax: +86-531-88382923 \\ (c) Ivyspring International Publisher. This is an open access article distributed under the terms of the Creative Commons Attribution (CC BY-NC) license \\ (https:// creativecommons.org/licenses/by-nc/4.0/). See http://ivyspring.com/terms for full terms and conditions.
}

Received: 2017.04.10; Accepted: 2017.08.07; Published: 2017.09.19

\begin{abstract}
Objective: The purpose of this study was to provide an insight into the biological effects of knockdown Yes-associated protein (YAP) on the proliferation and apoptosis of human periodontal ligament stem cells (h-PDLSCs). Methods: Immunofluorescence and Western blot were used to evaluate Hippo-YAP signaling expression level. Enhanced green fluorescence protein lentiviral vector was constructed to down-regulate YAP in h-PDLSCs. Real-time quantitative reverse transcription polymerase chain reaction ( $q R T-P C R$ ) and Western blot were used to detect the interfering efficiency of YAP expression. The proliferation activity was detected by EdU staining. Analysis of apoptosis in h-PDLSCs was done through Annexin V-APC staining, while cell cycle analysis was detected by flow cytometry. Cellular senescence was analyzed by $\beta$-galactosidase activity detection. The expression of elements in signaling pathways related with proliferation and apoptosis was detected by Western blot. Results: YAP was located in nucleus and cytoplasm. After the lentivirus transfection, the expression of YAP mRNA and protein was significantly reduced $(\mathrm{P}<0.001)$. When YAP was knocked down, the proliferation activity of h-PDLSCs was inhibited; the early \& late apoptosis rates increased; the proportion of cells in Gl phases increased $(P<0.05)$, while that in $G 2$ and $S$ phase decreased $(P<0.05)$; cellular senescence was accelerated $(\mathrm{P}<0.01)$; ERK and its target proteins $\mathrm{P}-\mathrm{P} 90 \mathrm{RSK}$ and $\mathrm{P}-\mathrm{MEK}$ were reduced while $\mathrm{Bcl}-2$ family members increased. Conclusion: Knockdown of YAP inhibits the proliferation activity and induces apoptosis of h-PDLSCs with the involvement of Hippo pathway and has a crosstalk between Erk and $\mathrm{Bcl}-2$ signaling pathways.
\end{abstract}

Key words: Yes-associated protein (YAP); human periodontal ligament stem cells (h-PDLSCs); proliferation; apoptosis.

\section{Introduction}

The periodontal ligament is generally defined as a self-renewal system, similar to a kind of undifferentiated cells which possess the capability of self-renewing and multi-differentiation [1]. Human periodontal ligament stem cells (h-PDLSCs) are regarded as the "cornerstone" of periodontal tissue regeneration and reconstruction. In the field of regenerative dentistry, the application of h-PDLSCs is the research hotspot in periodontal therapy. It has been confirmed that h-PDLSCs are a kind of key cells which can maintain dynamic balance and repair the damage of periodontal tissue [2]. Furthermore, they can also form new periodontal tissue structure in vivo experiments [3]. The biological basis of tissue 
regeneration is the proliferation, differentiation and orderly regulation of stem cells. When exposed to outside stimulus or disease, stem cells can continuously proliferate and differentiate to help tissue repair and regenerate. Stem cells can transform to any kinds of cells in the body, but how to preserve the ability and when it "decide" to abandon this state and transform into specific cells still remain a mystery until now. If the two problems were solved, the application of stem cells in regenerative medicine will be promising. And tissue regeneration therapy requires a sufficient number of seed cells, but it takes 2-4 weeks to amplify from the primary cell to the application order of $10^{7}-10^{8}[3,4]$, and it is prone to cell aging, dry down, then losing totipotency in the process of stem cell expansion. Currently, the main research direction in periodontal tissue engineering is to achieve the rapid proliferation of seed cells, maintain the potential of multi-directional differentiation, prevent the aging, maintain the activity of stem cells, differentiate into functional cells timely and promote the regeneration of periodontal tissue. Adult stem cell maintenance is required to sustain long-term preservation of tissue homeostasis. In animals, stem cells divide to new cells which can grow and take part in renewing tissues and organs. Understanding the biology of these cells is of the most importance for developing new treatments for a wide range of human diseases.

Hippo pathway is a newly discovered signaling network, which is evolutionarily and functionally conserved and has been shown to play a critical role in controlling organ size by regulating both cell proliferation and apoptosis [5-7]. Initially, this pathway was discovered in Drosophila melanogaster by mosaic genetic screens, which proved to be a powerful tool in the elucidation of this molecular signaling [8]. Yes-associated protein (YAP) and its paralogue transcriptional co-activator with PDZ binding motif (TAZ) shuttle between the cytoplasm and the nucleus and interact with transcription factors to regulate their activity [9]. Inhibition of the pathway promotes YAP/TAZ translocation to the nucleus, where they interact with transcriptional enhancer associate domain (TEAD) transcription factors and co activate the expression of target genes, promoting cell proliferation. As a Hippo signaling transcriptional co-activator, YAP plays pivotal roles in stem cell fate and organ size control. YAP has been shown to be a candidate oncogene in the development and progression of multiple human cancers [10-12]. Uncontrolled activity of YAP causes tissue overgrowth due to modulation of stem cell proliferation in multiple tissue and organs, including liver [13], intestine [13], brain [14], epidermis [15], muscle [16] and myocardium [17]. The expression patterns of YAP in the development of mouse incisor have been reported previously [18]. Their results have demonstrated the important relationship between YAP and stem cells proliferation. In addition, overexpression of YAP has an impact on tooth morphogenesis, enamel knot patterning, cells polarization and cells movement [19].

The present study aimed to investigate the effects of proliferation and apoptosis of YAP knockdown h-PDLSCs and also to explore the regulation mechanisms between this process to clarify the role of YAP in the regulation of proliferation in h-PDLSCs.

\section{Materials and Methods}

\section{Cell cultivation and identification}

h-PDLSCs were isolated and cultured as previously described $[20,21]$. The study protocol was approved by the Ethics Committee of School of Stomatology Shandong University (20151102), and written informed consent was obtained from each donor's parents in accordance with the Declaration of Helsinki. Periodontal ligament tissues were separated from root surface and were minced into pieces of small size $(1 \mathrm{~mm} \times 1 \mathrm{~mm} \times 1 \mathrm{~mm})$. The minced tissues were incubated with $3 \mathrm{mg} / \mathrm{ml}$ collagenase type I (Sigma) and $4 \mathrm{mg} / \mathrm{ml}$ dispase (Sigma) in a-MEM (Gibco) at $37{ }^{\circ} \mathrm{C}$ for $1 \mathrm{~h}$. Single cells in suspension were obtained by passing through a strainer (pore size: $70 \mu \mathrm{m}$ from BD Falcon Labware). Then the cells were seeded in $10 \mathrm{~cm}$ petri dishes containing a-MEM supplemented with 15\% FBS (Gibco), $2 \mathrm{mM}$ L-glutamine, $100 \mathrm{U} / \mathrm{ml}$ penicillin, and $100 \mathrm{mg} / \mathrm{ml}$ streptomycin (Gibco), and incubated at $37^{\circ} \mathrm{C}$ in $5 \% \mathrm{CO}_{2}$. Cells at passages P3-P5 were used for the following experiments.

The passage 3 cells were used to identify the stem cell properties. $1 \times 10^{6}$ cells were incubated with hMSC positive cocktail (CD90 FITC, CD105 PerCP-Cy5.5, CD73 APC, CD44 PE), hMSC positive isotype control cocktail (mIgG1 kFITC, mIgG1 кPerCP-Сy5.5, mIgG1 кAPC, mIgG2b кPE), HSCs positive cocktail (CD34 PE, CD11b PE, CD19 PE, CD45 PE, HLA-DR PE), PE hMSC negative isotype control cocktail (mIgG1кPE, mIgG2a кPE) respectively (BD Stemflow ${ }^{\mathrm{TM}}$ hMSC Analysis Kit, BD Bioscience, NJ, USA) and analyzed in a BD FACSCalibur flow cytometer (BD Biosciences, NJ, USA). Cells were seeded on 6-well culture plates at a density of $5 \times 10^{4}$ cells per well and induced in osteogenic and adipogenic induction medium respectively for 28 days and stained with Alizarin Red and Oil Red O. For chondrogenic induction, $2.5 \times 10^{5}$ 
cells were seeded in a culture tube to form a pellet culture. Alcian Blue was used to stain the tissue section after 28 days induction. All the induction media were bought from Cyagen Biosciences Inc. (Guangzhou, China).

\section{Virus packaging and transfection assay}

Lentivirus packaging cells were transfected with PGLV3-h1-GFP-puro vector (GenePharma, Shanghai, China) containing either the YAP knockdown (shYAP) or a negative control sequence (NC). h-PDLSCs were infected at approximately $70 \%$ confluence by the culture medium with $8 \mu \mathrm{g} / \mathrm{ml}$ polybrene. After $6 \mathrm{~h}$, the medium was changed to basal medium supplemented with 10\% FBS and cells were cultured for further assays. The efficiency for knockdown YAP was determined by Western blot and Real-time quantitative reverse transcription polymerase chain reaction (qRT-PCR) assays. YAP-shRNA Sequences are listed in table 1.

Table 1. YAP-shRNA Sequences

\begin{tabular}{ll}
\hline Yap-shRNA & \\
\hline shRNA-1 & 5'-GCAUCUUCGACAGUCUUCUTT-3' \\
shRNA-2 & 5'-GGUGAUACUAUCAACCAAATT-3' \\
NC & 5'-UUAUCUAGCUUGGUGGCAGTT-3' \\
\hline
\end{tabular}

\section{RNA isolation and qRT-PCR.}

Total RNA was prepared using TRIzol Reagent (Invitrogen) following manufacturer's instructions. Total RNA (1 $\mathrm{g})$ was subjected to reverse transcription to synthesize cDNA using the SuperScript ${ }^{\mathrm{TM}}$ II Reverse Transcriptase Kit (Invitrogen). For qRT-PCR, each reaction $(25 \mu \mathrm{L})$ consisted $1 \mu \mathrm{L}$ reverse transcription cDNA product and $100 \mathrm{nM}$ of each primer. qRT-PCR reactions were then performed as follows: one cycle of $95^{\circ} \mathrm{C}$ for 30s, followed by 40 cycles of $95^{\circ} \mathrm{C}$ for $5 \mathrm{~s}, 60^{\circ} \mathrm{C}$ for $20 \mathrm{~s}$. qRT-PCR was carried out in LightCycler®480II, and changes in gene expression were calculated using the delta-delta CT method. GAPDH was used to normalize gene expression in each sample in different groups. The primers used for qRT-PCR are listed in table 2.

\section{Western blot analysis}

Cells were collected and lysed in RIPA buffer in the presence of protease inhibitors. Protein concentrations were determined by the BCA method using chemiluminescence reader ImageQuant LAS4000 (GE, USA). 20 $\mu$ g of protein were separated by $10 \%$ SDS-PAGE and electroblotted to a PVDF membrane using a wet transfer apparatus (Bio-Rad, Hercules, CA, USA). After blocking with 5\% nonfat milk, the membranes were incubated overnight at $4^{\circ} \mathrm{C}$ with the primary antibodies, followed by labeling with the secondary antibody. Protein bands were visualized with enhanced chemiluminescence (Millipore). Protein levels were analyzed by ImageJ software. GAPDH was used as the endogenous control and the control cells were cultured in the complete medium without sh-RNA.

\section{EdU incorporation assay}

Cell proliferation was assessed using an EdU Apollo DNA in vitro kit (RIBOBIO) following the manufacturer's instructions. h-PDLSCs were seeded at a density of $1 \times 10^{4} \mathrm{cell} / \mathrm{cm}^{2}$ in 24-well plates and incubated for $24 \mathrm{~h}$ in normal growth medium. Cells were treated with $50 \mathrm{mM}$ EdU for $2 \mathrm{~h}$, then were fixed with $4 \%$ paraformaldehyde for $15-20 \mathrm{~min}$ at room temperature. The cells were then incubated with $2 \mathrm{mg} / \mathrm{ml}$ glycine for 10min followed by washing with PBS. After that, the cells were permeated with $100 \mathrm{ml} /$ well permeabilization buffer $(0.5 \%$ Triton X-100 containing PBS) and incubated with $100 \mathrm{ml}$ of 1X Apollo solution for $30 \mathrm{~min}$ at room temperature in the dark. Subsequently, cells were incubated with $100 \mathrm{ml}$ of $1 \mathrm{X}$ Hoechst 33342 solution for $30 \mathrm{~min}$ at room temperature in the dark. Afterwards, the samples were observed under fluorescence microscope.

\section{Immunofluorescence study}

The cells were plated on coverslips and cultured for $1 \mathrm{~d}$. After fixed in $4 \%$ paraformal-dehyde (PFA) for $30 \mathrm{~min}$ at room temperature, the cells were incubated in $0.1 \%$ Triton X-100 for $10 \mathrm{~min}$ and washed with PBS. Then cells were blocked in Blocking Buffer for $60 \mathrm{~min}$ and incubated overnight at $4^{\circ} \mathrm{C}$ with primary antibodies (1:100, CST, USA) diluted in blocking solution. After that, cells were washed in PBS for 3 times and incubated in fluorochrome-conjugated secondary antibody (1:500, CST, USA) for $1 \mathrm{~h}$ at room temperature in the dark. Finally, cells were counterstained with DAPI $(1 \mu \mathrm{g} / \mathrm{ml}, \mathrm{CST}$, USA) for 5 min and observed under a fluorescence microscope.

Table 2. Primers for Quantitative PCR

\begin{tabular}{lll}
\hline GENE & Forward primer 5'-3' & Reverse primer 5'-3' \\
\hline YAP & 5'-AATGACGACCAATAGCTCAGATCC-3' & 5'-CACTGTAGCTGCTCATGCTYAGTCC-3 \\
GAPDH & 5'-AGAAGGCTGGGGCTCA7ITITIG-3' & 5'-AGGGGCCATCCACAGTCTTC-3' \\
\hline
\end{tabular}




\section{Flow-cytometry analysis for cell cycle}

$5 \times 10^{5}$ cells were washed with cold PBS and fixed overnight with $70 \%$ cold ethanol at $-20^{\circ} \mathrm{C}$. The fixed cells were centrifuged at $1200 \mathrm{~g}$ for $1 \mathrm{~min}$, and washed with PBS twice. After that, the cell plates were resuspended with $200 \mu \mathrm{l} \mathrm{RNase} \mathrm{A}(1 \mathrm{mg} / \mathrm{ml})$ at $37^{\circ} \mathrm{C}$ for $10 \mathrm{~min}$, followed by the addition of $300 \mu \mathrm{l}$ propidium iodine (PI, $100 \mu \mathrm{l} / \mathrm{ml}$ ) to stain the DNA of cells in the dark. After $20 \mathrm{~min}$ incubation at room temperature, the DNA contents of the cells were analyzed in a FACScan flow cytometer (Becton Dickinson, Franklin Lakes, NJ, USA) and the data was analyzed by ModFitLT V2.0 software (Becton Dickinson).

\section{Apoptosis assay}

Cells were collected, and the translocation of phosphatidylserine in cells was detected using the Annexin-V-APC staining kit (Sungene Biotech Co, Ltd.). Briefly, cells were suspended in $500 \mu \mathrm{L}$ of binding buffer and incubated at room temperature in the dark for 10min after labeled with $5 \mu \mathrm{L}$ of Annexin $\mathrm{V}$-fluorescein APC. The cells were vortexed and incubated for 10min in room temperature in the dark. Then $5 \mu \mathrm{L}$ 7-AAD solution was added and cells were incubated for $5 \mathrm{~min}$ in room temperature, in the dark. The stained cells were then analyzed by flow cytometry. The data was analyzed by WinMDI V2.9 software (The Scripps Research Institute, San Diego, CA, USA).

\section{Senescence Associated Beta galactosidase staining}

Cells were washed in PBS and then fixed in a solution containing $4 \%$ paraformaldehyde for $20 \mathrm{~min}$. The cells were then washed in PBS and water and stained in a beta galactosidase solution. The cells were stained for $24 \mathrm{~h}$ in $37^{\circ} \mathrm{C}$ without carbon dioxide. Then staining cells were counted in 6 randomly selected high-power microscopic fields $(\times 100)$ per filter under microscopy.

\section{Statistical analysis}

The student's two-tailed t-test was used to determine statistical differences between treatment and control values. All data were presented as the mean $\pm S D$ of three independent experiments. Data was analyzed by one-way analysis of variance or t-test by using the SPSS software (SPSS 19.0). Differences were considered statistical significant when $p<0.05$.

\section{Results}

\section{Characterization of hPDLSCs}

h-PDLSCs exhibited typical spindle-like morphology (Fig. 1A). Flow cytometry analysis showed that h-PDLSCs were negative to HSCs negative cocktail (CD11b, CD19, CD34, CD45, HLA-DR), but highly expressed positive hMSC positive cocktail (CD73, CD90, CD105, CD44) (Fig. 1B). These results indicated that h-PDLSCs shared the similar phenotype with MSCs. The formation of mineralized nodules, lipid droplets and blue proteoglycans after induction indicated the cells possessed multipotentiality properties (Fig. 1C, D and $\mathrm{E})$.
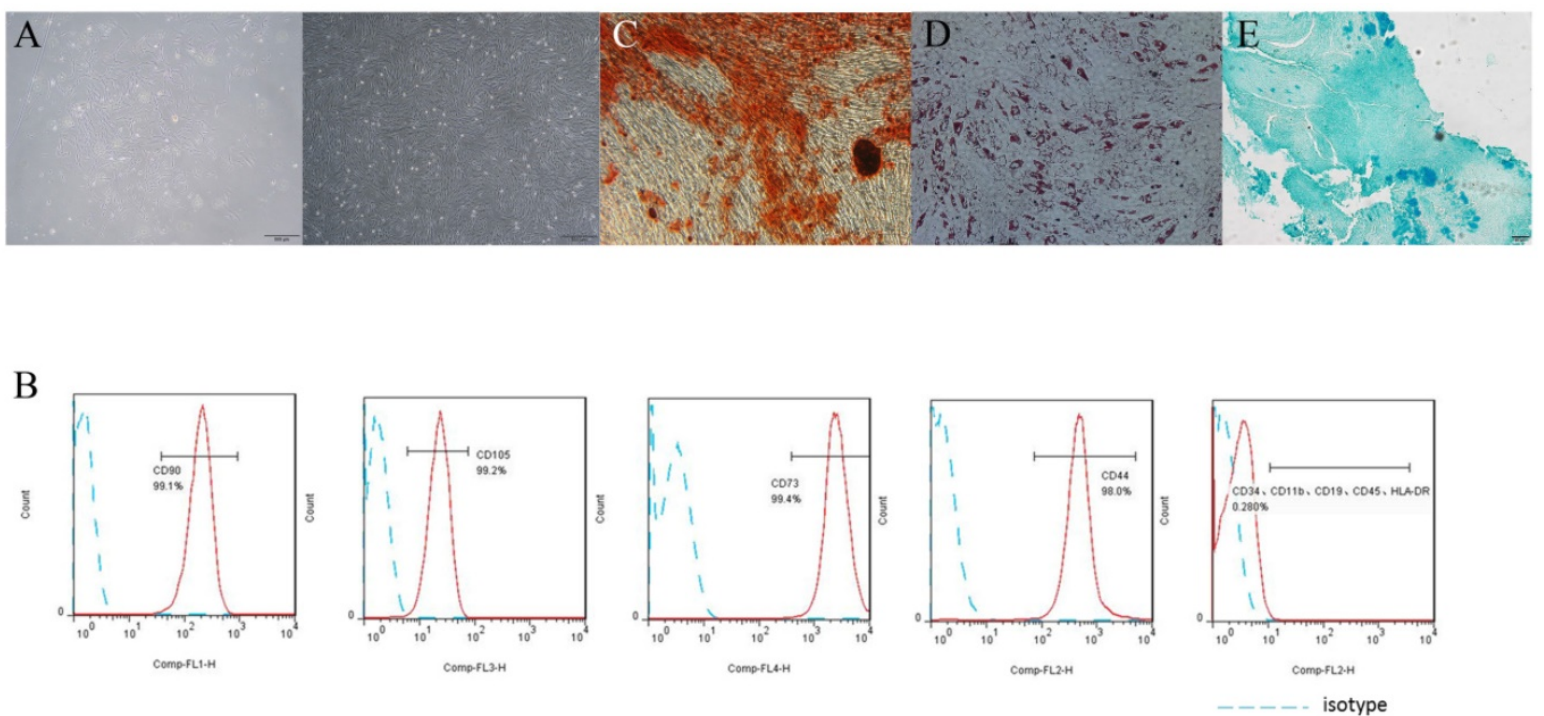

Figure 1. PDLSCs characteristics assay (A) The morphology of PDLSCs, left: primary culture, right: passages P4. (B) Flow cytometry analysis showed that PDLSCs expressed CD73, CD44, CD90 and CD105, did not express CD34, CD1 lb, CD19, CD45 and HLA-DR. (C) Cells cultured in osteogenic induction medium for 4 weeks, Stained with Alizarin Red. (D) Cells cultured in adipogenic induction medium for 4 weeks, Stained with Oil Red O. (E) Cells cultured in chondrogenic

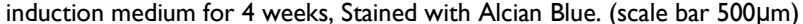




\section{Hippo-YAP signal expression in h-PDLSCs}

h-PDLSCs collected from 5 donors were used to investigate the expression of Hippo-YAP core elements. SAV1, Mst1, Mst2, MOB1(E1N9D), phospho-MOB1 (Thr35), LATS1, YAP, phospho-YAP (Ser127), phospho-YAP (ser397), TAZ were detected in h-PDLSCs. There was a slight difference in the expression pattern of the core elements among different h-PDLSCs sources (Fig. 2A). YAP was detected either in the cytoplasmic or in the nuclear of hPDLSCs by immunofluorescent. (Fig. 2B)

\section{Detection of YAP interference efficiency}

There was a significant reduction of YAP mRNA expression in shYAP when compared with negative control group $(\mathrm{P}<0.001)$ (Fig. 3A). Western blot results showed that YAP protein expression in shYAP groups were significantly lower $(\mathrm{P}<0.001)$ (Fig. 3B). Immunofluorescent staining results showed that YAP protein expression in shYAP groups were significantly lower (Fig. 3C). These results demonstrated that the YAP interference efficiency was higher and more stable in group shYAP. Therefore, shYAP were selected as experimental group in the following experiments.
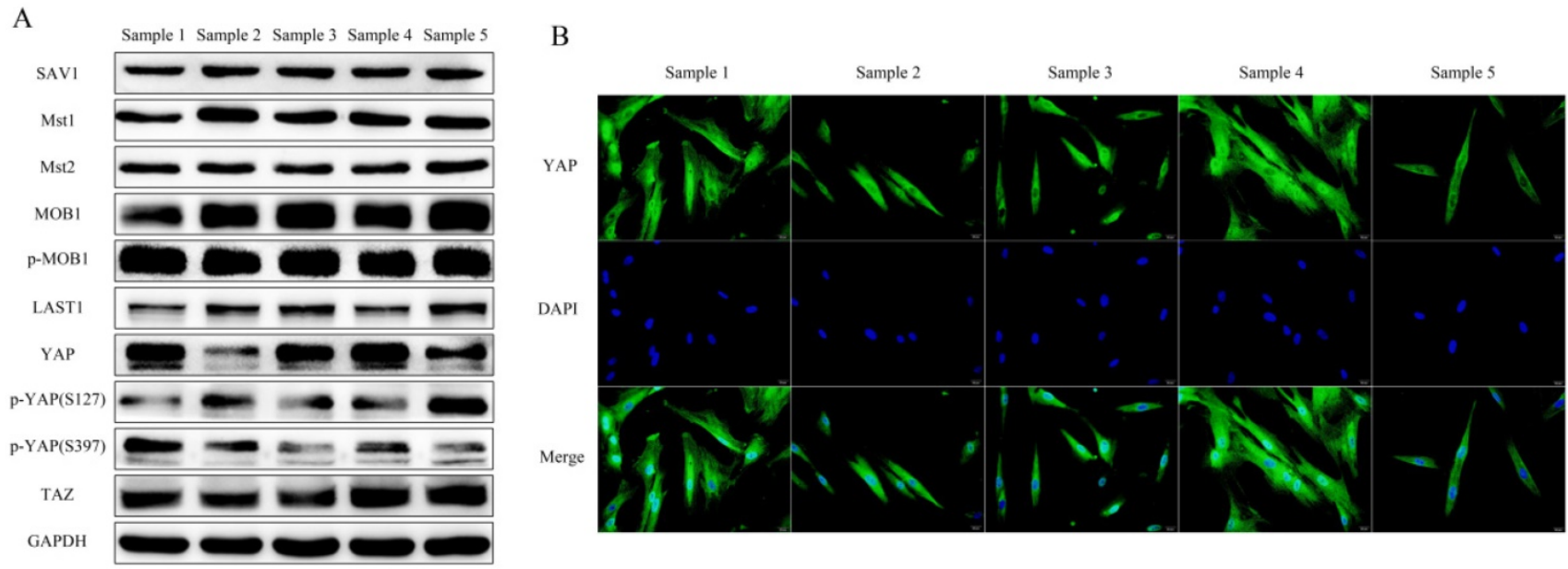

Figure 2. Hippo-YAP signal pathway expression in PDLSCs (A) Western blot detected the Hippo-YAP signal pathway expression in different samples of PDLSCs, include SAV1, Mst1, Mst2, MOB1 (EIN9D), phospho-MOB1 (Thr35), LATS1, YAP, phospho-YAP (Ser 127), phospho-YAP (ser397) and TAZ was examined. GAPDH serves as a loading control. (B) Immunofluorescent staining showed the YAP located in nucleus or cytoplasm of PDLSCs. The nucleus were counterstained with DAPI. (scale bar $20 \mu \mathrm{m}$ )

A

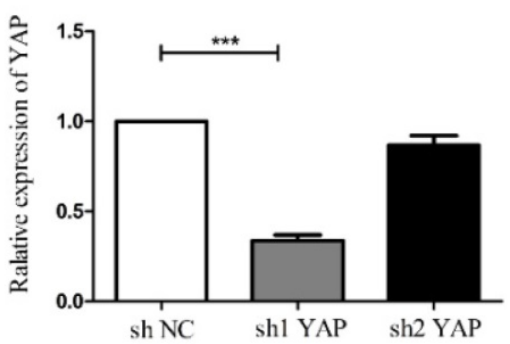

B

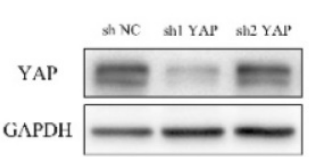

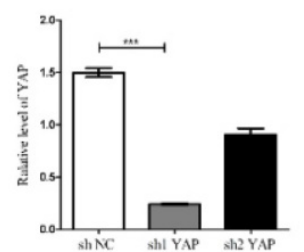

$\mathrm{C}$

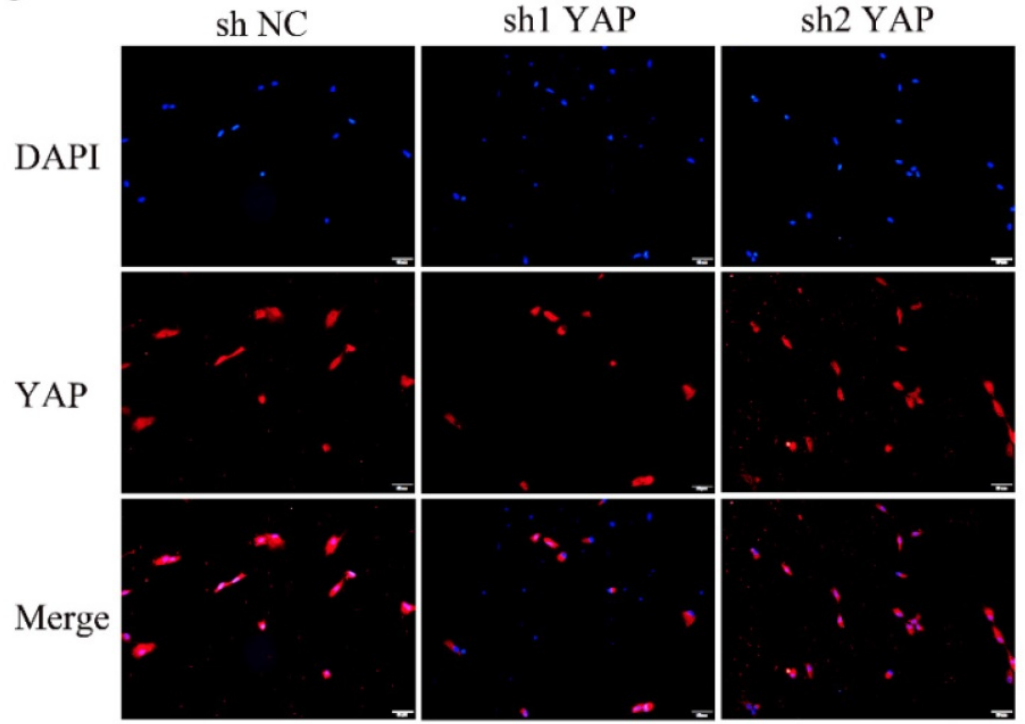

Figure 3. Detection of YAP interference efficiency (A)There was a significant reduction of YAP mRNA expression in shIYAP when compared with negative control group $(* * * \mathrm{P}<0.001)$ (Fig. 3A). Western blot results showed that $\mathrm{YAP}$ protein expression in shlYAP groups were significantly lower $(* * * P<0.001)($ Fig. $3 \mathrm{~B})$. Immunofluorescent staining results showed that YAP protein expression in sh1YAP groups were significantly lower (Fig. 3C) 


\section{Knock down YAP inhibited h-PDLSCs proliferation}

EdU result showed that the proliferation rate of shYAP reduced markedly after transfection compared with NC group $(\mathrm{P}<0.01)$ (Fig.4A). The expression of p-C-Raf338 and p-MEK, which take part in the phosphorylation of Erk, was inhibited when YAP was knocked down. At the same time, the protein levels of p-ERK and its downstream proteins p-p90RSK and p-MSK were reduced with the interfering of YAP.

\section{Knock down YAP induced $h$-PDLSCs apoptosis}

To determine whether YAP knockdown increased the apoptosis rate in h-PDLSCs, the percentage of apoptosis was examined using flow cytometry. The percentage of early apoptotic cells was $0.348 \pm 0.045 \%$ in the shYAP group, $0.165 \pm 0.030 \%$ in the NC group, while the percentage of late apoptotic cells were $3.003 \pm 0.295 \%$ in the shYAP group and $1.218 \pm 0.098 \%$ in the NC group. The percentage of early and late apoptotic cells in shYAP group were significantly increased after YAP knockdown compared with NC group ( $p<0.001)$ (Fig. 5A).

As for apoptosis related proteins including Caspase3 and Bcl-2 family members (Bak, Bax, Bad, Bid and Bik), their expression levels increased separately after knocking down YAP (Fig. 5B).

\section{Knock down YAP induced GI/S arrest}

Flow-cytometry analysis results showed that h-PDLSCs were mostly in G0 / G1 phase of the cell cycle, which is slow periodicity. When compared with NC group, the proportion of cells in G0/G1 phase increased evidently $(\mathrm{P}<0.05)$, while that in $\mathrm{S}$ and $\mathrm{G} 2 / \mathrm{M}$ phase decreased $(\mathrm{P}<0.05)$ in shYAP group (Fig. 6A).

Both cyclin dependent kinases (CDKs) responsible for G1/S phase transition (CDK4/6-Cyclin D1 and CDK2-Cyclin E2) and CDK inhibitors (p27 and p21) were up-regulated when YAP was knocked down (Fig. 6B).
A

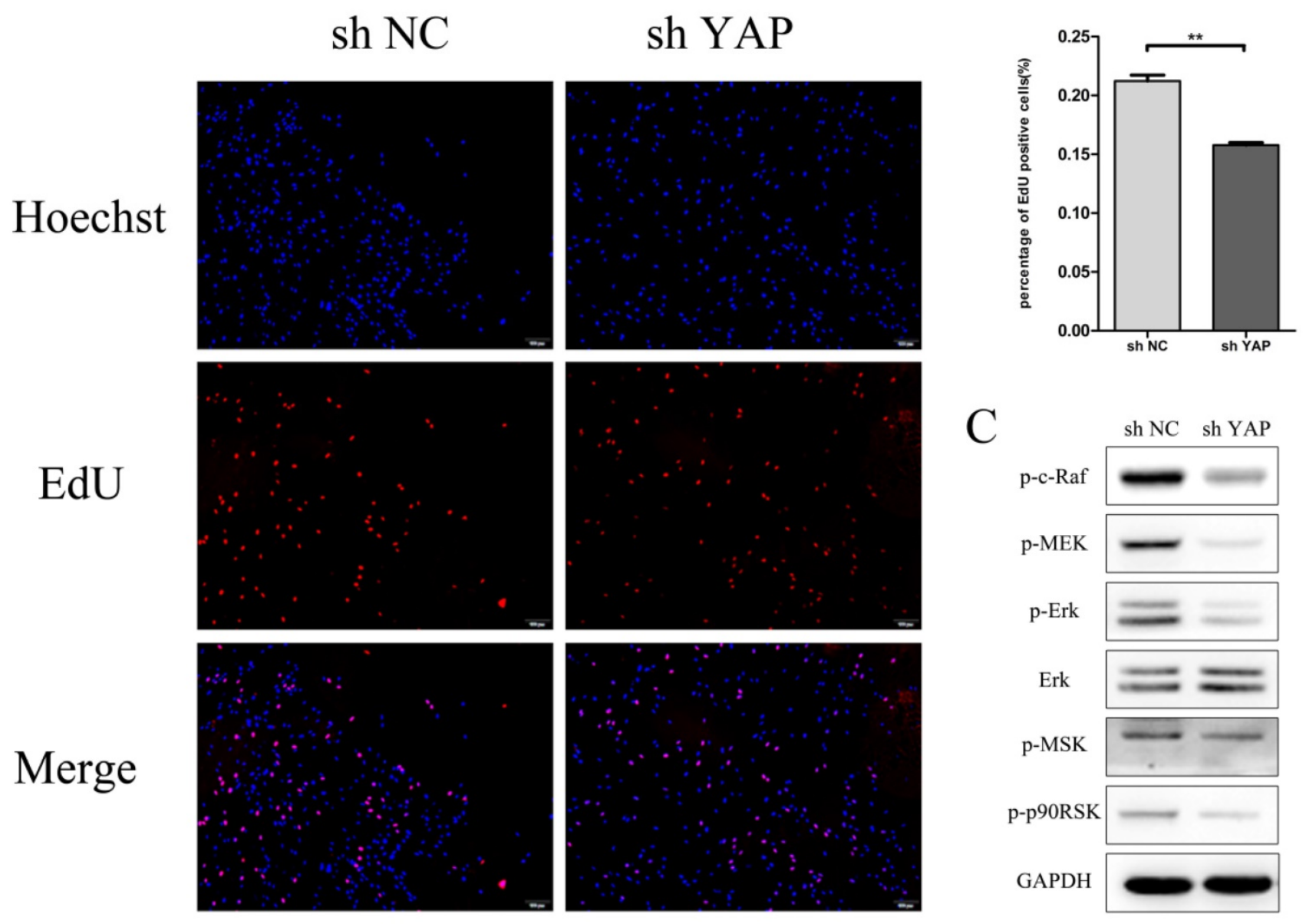

Figure 4. Knock down YAP inhibited PDLSCs proliferation (A) EdU staining was used to evaluate the proliferation ability of PDLSCs (B)Data showed that PDLSCs proliferation reduce markedly after transfect shYAP compared with NC group (**P<0.01) (C) The expression of p-C-Raf338, p-MSK1, P-P90RSK and P-MEK was inhibited when YAP was knocked down. (scale bar $100 \mu \mathrm{m}$ ) 
A

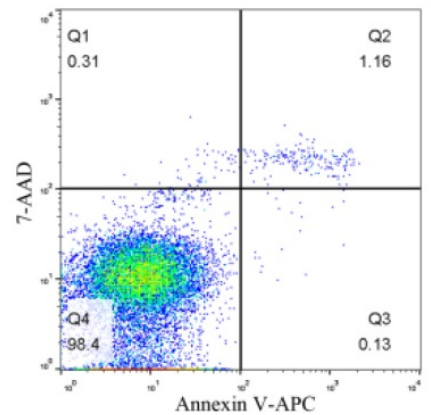

sh NC

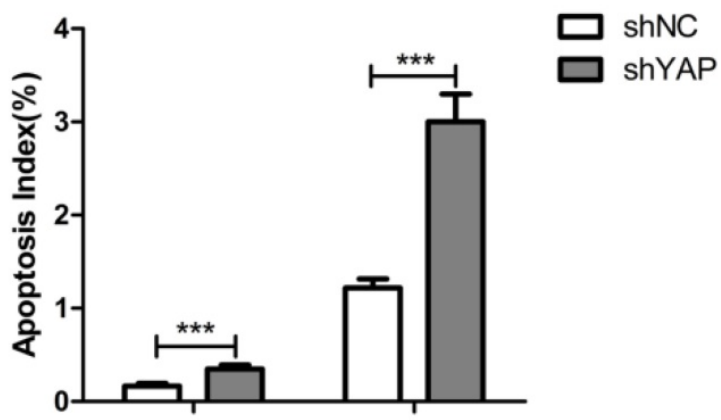

B

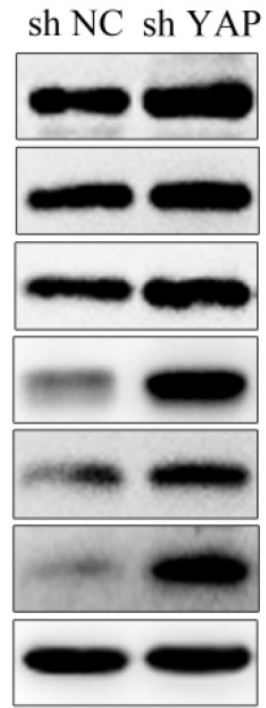

Caspase 3

Bak

Bax

$\mathrm{Bad}$

Bid

Bik

GAPDH

Figure 5. Knock down YAP induced PDLSCs apoptosis (A) Cell-cycle analysis was performed using the standard method, apparently, the early and late apoptosis rate in shYAP group was obviously increased (***p<0.001), (B) Caspase3 and Bcl-2 family members (Bak, Bax, Bad, Bid and Bik), their expression levels increased separately after knock down YAP.

A
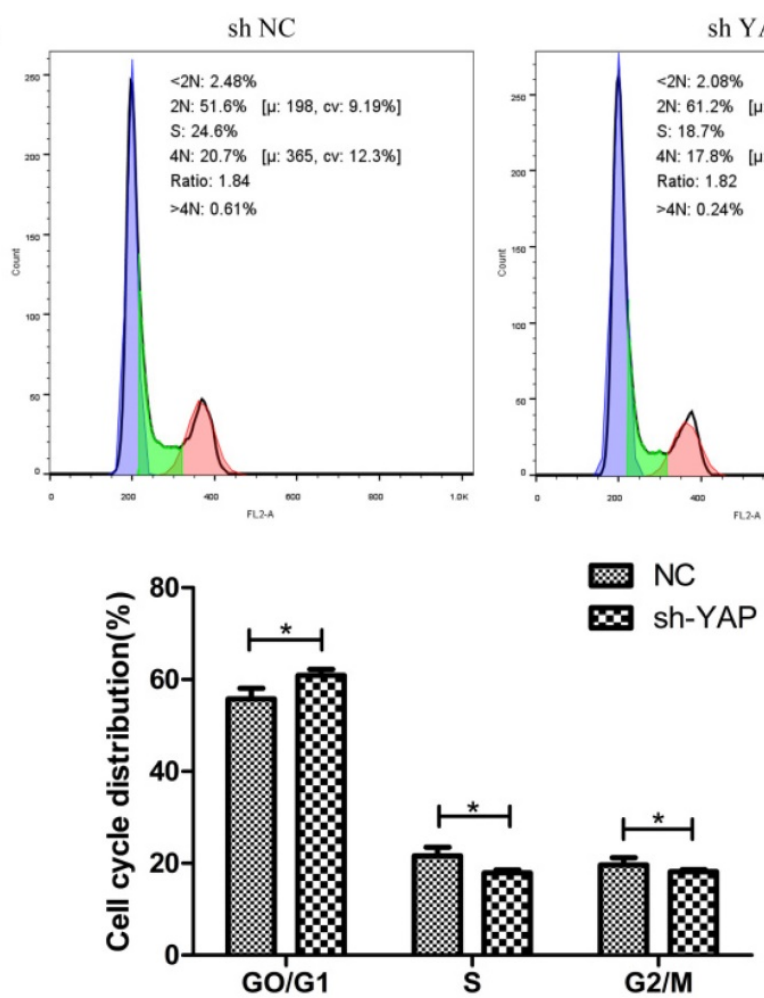

$\mathrm{B}$
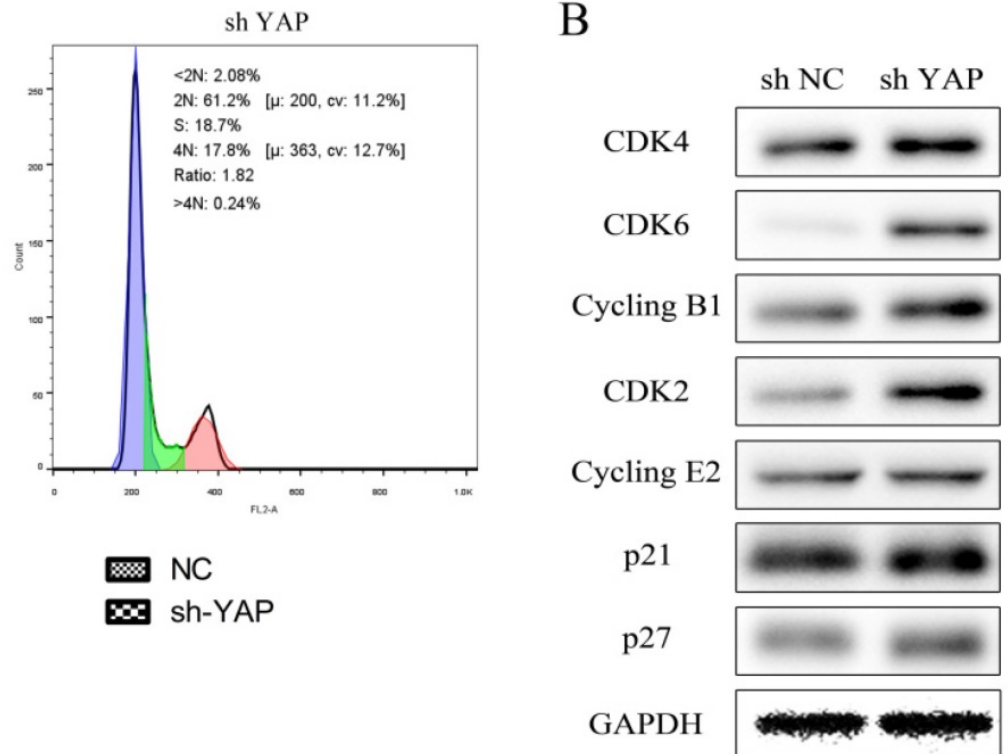

Figure 6. Knock down YAP induced G1 arrest (A) The distribution of cell cycle in shYAP group changed, the proportion of cells in G1 phase increased evidently $(* \mathrm{P}<0.05)$, and cells in S and G2 phase was markedly decreased (*P < 0.05). (B) Both cyclin dependent kinases (CDKs) responsible for G1/S phase transition (CDK4/6-Cyclin D1 and CDK2-Cyclin E2) and CDK inhibitors (p27 and p21) were up-regulated when YAP was knocked down. 


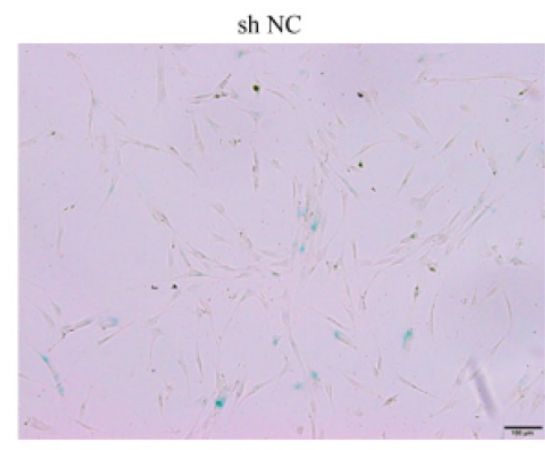

sh YAP

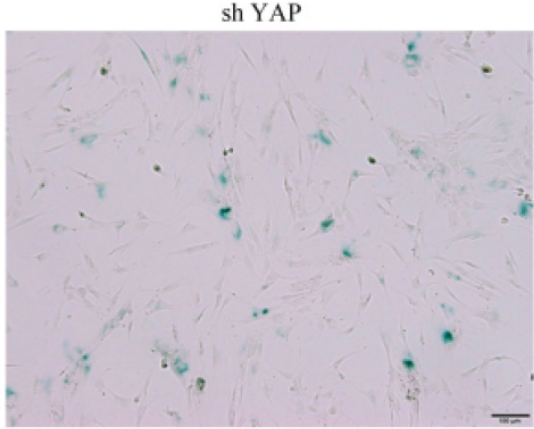

Cell counting results of Beta - galactose glucoside enzyme staining

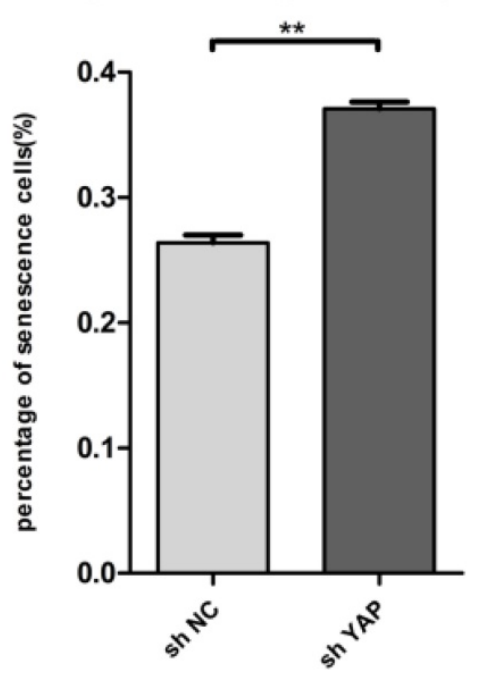

Figure 7. Knock down YAP induced cellular senescence (A) Senescence associated beta galactosidase staining indicate shYAP group showed higher senescence cells rate compared with NC group. (B) Date showed higher senescence cells rate in shYAP group compared with NC group (**P<0.01). (scale bar $100 \mu \mathrm{m})$

\section{Knock down YAP induced cellular senescence}

To know the effect of YAP on the senescence of h-PDLSCs, we used the senescence associated beta galactosidase staining assay to detect the senescence of h-PDLSCs (Fig. 7A). shYAP group showed higher senescence cells rate compared with NC group (Fig. $7 \mathrm{~B})(\mathrm{P}<0.01)$. The findings indicated that knock down $\mathrm{YAP}$ induced the senescence of h-PDLSCs.

\section{Discussion}

In the present study, we identified YAP, a downstream effector of the Hippo pathway, as a stem cell specific marker required for homeostatic growth of the PDLSCs. After we knocked down the YAP expression with sh-RNA, EdU result revealed that the activity of cell proliferation reduced, the cell growth rate slowed down and the cell growth was suppressed persistently. Cell cycle analysis in our experiments suggested that the proportion of cells in G1 phase increased while the G2 and S phase obviously decreased. The latest study finds that YAP has a crucial effect on the regulation of cell cycle in endothelial cells, which means knockout YAP gene results to the proportion of cells in G1 phase increase and $S$ phase decrease. However, when YAP gene is knocked out on HeLa cells, the cell cycle distribution has no change [22]. So we speculate that the regulation mechanism of YAP in cell cycle varies in different cells.
Erk is a member of mitogen-activated protein kinase (MAPK), and Erk signaling pathway has been proved to take part in the regulation of cell proliferation [23-25]. In our study, the expression of p-C-Raf338 and p-MSK1, which take part in the phosphorylation of Erk, was inhibited when YAP was knocked down, and the protein levels of ERK and its downstream proteins P-P90RSK and P-MEK, were also reduced. Since P-P90RSK and P-MEK play roles in proliferation regulation [26, 27], we suggested that crosstalk between Erk and hippo signaling pathway affected h-PDLSCs proliferation.

In our study, the apoptosis rate of h-PDLSCs apparently increased after YAP gene was interfered. At the same time, the expression levels of Bcl-2 family members (Bak, Bax, Bad, Bid and Bik) increased when YAP was knocked down. This result reflected the association between Bcl-2 signaling pathways and hippo signaling pathway, and YAP could regulate the apoptosis rate of h-PDLSCs through Bcl-2 signaling pathways.

YAP was also associated with cell senescence, while the effects were controversial. For example, some scholars found that the activation of an ATM-YAP-PML-p53 axis could accelerate cellular senescence in Werner syndrome -derived fibroblasts [28], and YAP/TAZ activation in hepatocytes induced massive p53-dependent cell senescence/death [29], while others proved that down-regulation of YAP in 
IMR90 tumor cells increased cells senescence [30], which was consistent with our experiments---knock down YAP induced senescence in h-PDLSCs. Thus, the regulation mechanism of YAP in cell senescence is also quite different in various cells.

In fact, our experiment results were consonant with previous studies. Many researches have proved that Hippo pathway in mammalian is involved in cell proliferation, apoptosis, migration and differentiation [31]. As the switch protein, YAP plays a central role in the Hippo signaling pathway. In the process of normal growth and development, YAP can bind transcription factor TEAD to promote the expression of downstream target genes, so as to accelerate cells growth and inhibit cells apoptosis. Undifferentiated progenitor cells get obvious expansion because of the over expression of YAP [13], both multifunctional knockout and RNA interference of YAP reduce the multi-differentiated potentiality of stem cells significantly, and the expression of genes Oct4 and Sox2 that maintain stem cells characteristics decrease greatly as well [32]. Recent investigations demonstrate that YAP gene is involved in the self-renewal of stem cells $[33,34]$.The study of neural progenitor cells indicates that over expression of YAP could not only inhibit its differentiation, but also enhance its mass proliferation; YAP is also expressed in intestinal epithelial precursor cells located in the small intestine of normal mice, which means YAP takes part in regulating tissue-specific precursor cells, furthermore, YAP over expression can regulate precursor cells proliferation significantly, while inhibits precursor cells from differentiating into mature terminal cells [13].Thus, YAP has a significant effect on maintaining self-renewal and differentiation of stem cells as a gene transcriptional co-activator. And the action of promoting proliferation of YAP is closely related to TEAD, moreover, the activation of YAP/TEAD facilitates expression of related genes such as cyclin D1, while it inhibits stem cells exiting the cell cycle and cells apoptosis, which is conducive to the proliferation of stem cells. As an important kind of stem cells, h-PDLSCs were proved to participate in maintaining the balance of periodontal ligament through orient migration and differentiation [35]. Since the regulation mechanism of proliferation, apoptosis and senescence in h-PDLSCs remains uncertain until now, we suggested that Hippo pathway took part in this regulation process. Our results proved that knocking down YAP induced the apoptosis and inhibited the proliferation of h-PDLSCs.

Above all, our preliminary results indicate that YAP can promote proliferation and inhibit the apoptosis of h-PDLSCs, knock down YAP induced senescence in h-PDLSCs, which may offer a new idea for the regeneration of periodontal tissues based on stem cells. Nevertheless, the regulation mechanism of YAP is still unclear, and further investigations are needed to elucidate the other biological changes of h-PDLSCs after YAP is up-regulated or down-regulated.

\section{Conclusions}

In this article, we mainly summarize that knockdown YAP expression inhibits the proliferation activity by inducing apoptosis, cell senescence and cell-cycle arrest of h-PDLSCs. Hippo-YAP signaling pathway takes part in regulating biological behaviors of h-PDLSCs and has crosstalk between Erk and Bcl-2 signaling pathways.

These results contribute to the understanding of the mechanism of YAP regulating the proliferation and apoptosis of h-PDLSCs and we look forward to discovering the mechanism.

\section{Acknowledgements}

This work was supported by grants from the National Natural Science Foundation of China (Grant No. 81300885 and 81402150), Shandong Provincial key research and development program (Grant No: 2015GSF118122, 2015GSF118183, 2016GSF201115 and 2016GSF201220 and 2017GSF18117), China Postdoctoral Science Foundation (Grant No: 2017M610432), Young Scholars Program of Shandong University (Grant No: 2015WLJH53) and the Construction Engineering Special Fund of Taishan Scholars (Grant No: ts201511106).

\section{Competing Interests}

The authors have declared that no competing interest exists.

\section{References}

1. Seo BM, Miura M, Gronthos S, Bartold PM, Batouli S, Brahim J, Young M, Robey PG, Wang CY, Shi S: Investigation of multipotent postnatal stem cells from human periodontal ligament. Lancet 2004, 364(9429):149-155.

2. Gronthos S, Mrozik K, Shi S, Bartold PM: Ovine periodontal ligament stem cells: isolation, characterization, and differentiation potential. Calcif Tissue Int 2006, 79(5):310-317.

3. Ding G, Liu Y, Wang W, et al. Allogeneic periodontal ligament stem cell therapy for periodontitis in swine. Stem Cells. 2010; 28: 1829-38.

4. Lee JS, Hong JM, Moon GJ, et al. A long-term follow-up study of intravenous autologous mesenchymal stem cell transplantation in patients with ischemic stroke. Stem Cells. 2010; 28: 1099-106.

5. Zhang L: Control of growth and beyond: a special issue on Hippo signaling. Acta biochimica et biophysica Sinica 2015, 47(1):1.

6. Halder G, Johnson RL: Hippo signaling: growth control and beyond. Development 2011, 138(1):9-22.

7. Tremblay AM, Camargo FD: Hippo signaling in mammalian stem cells. Seminars in cell \& developmental biology 2012, 23(7):818-826.

8. Santucci M, Vignudelli T, Ferrari S, Mor M, Scalvini L, Bolognesi ML, Uliassi E, Costi MP: The Hippo Pathway and YAP/TAZ-TEAD Protein-Protein Interaction as Targets for Regenerative Medicine and Cancer Treatment. Journal of medicinal chemistry 2015, 58(12):4857-4873.

9. Moroishi T, Park HW, Qin B, Chen Q, Meng Z, Plouffe SW, Taniguchi K, Yu FX, Karin M, Pan D et al: A YAP/TAZ-induced feedback mechanism regulates Hippo pathway homeostasis. Genes \& development 2015, 29(12):1271-1284. 
10. Yoshikawa K, Noguchi K, Nakano $\mathrm{Y}$, Yamamura $\mathrm{M}$, Takaoka K, Hashimoto-Tamaoki T, Kishimoto H: The Hippo pathway transcriptional co-activator, YAP, confers resistance to cisplatin in human oral squamous cell carcinoma. International journal of oncology 2015, 46(6):2364-2370.

11. Zhang X, George J, Deb S, Degoutin JL, Takano EA, Fox SB, group AS, Bowtell DD, Harvey KF: The Hippo pathway transcriptional co-activator, YAP, is an ovarian cancer oncogene. Oncogene 2011, 30(25):2810-2822.

12. Yimlamai D, Fowl BH, Camargo FD: Emerging evidence on the role of the Hippo/YAP pathway in liver physiology and cancer. Journal of hepatology 2015.

13. Camargo FD, Gokhale S, Johnnidis JB, Fu D, Bell GW, Jaenisch R, Brummelkamp TR: YAP1 increases organ size and expands undifferentiated progenitor cells. Curr Biol 2007, 17(23):2054-2060.

14. Cao X, Pfaff SL, Gage FH: YAP regulates neural progenitor cell number via the TEA domain transcription factor. Genes \& development 2008, 22(23):3320-3334.

15. Schlegelmilch K, Mohseni M, Kirak O, Pruszak J, Rodriguez JR, Zhou D, Kreger BT, Vasioukhin V, Avruch J, Brummelkamp TR et al: Yap1 acts downstream of alpha-catenin to control epidermal proliferation. Cell 2011, 144(5):782-795.

16. Watt KI, Turner BJ, Hagg A, Zhang X, Davey JR, Qian H, Beyer C, Winbanks CE, Harvey KF, Gregorevic P: The Hippo pathway effector YAP is a critical regulator of skeletal muscle fibre size. Nature communications 2015, 6:6048.

17. von Gise A, Lin Z, Schlegelmilch K, Honor LB, Pan GM, Buck JN, Ma O Ishiwata T, Zhou B, Camargo FD et al: YAP1, the nuclear target of Hippo signaling, stimulates heart growth through cardiomyocyte proliferation but not hypertrophy. Proc Natl Acad Sci U S A 2012, 109(7):2394-2399.

18. Li L, Kwon HJ, Harada H, Ohshima H, Cho SW, Jung HS: Expression patterns of ABCG2, Bmi-1, Oct-3/4, and Yap in the developing mouse incisor. Gene expression patterns : GEP 2011, 11(3-4):163-170.

19. Liu M, Zhao S, Wang XP: YAP overexpression affects tooth morphogenesis and enamel knot patterning. Journal of dental research 2014, 93(5):469-474.

20. Wen Y, Lan J, Huang H, Yu M, Cui J, Liang J, Jiang B, Xu X: Application of eGFP to label human periodontal ligament stem cells in periodontal tissue engineering. Archives of oral biology 2012, 57(9):1241-1250.

21. Jiang B, Wen Y, Huang H, Cui J, Liang J, Ma X, Lan J, Xu X: [Study of labeling human periodontal ligament stem cells with enhanced green fluorescent protein by lentivirus vector infection]. Hua xi kou qiang yi xue za zhi $=$ Huaxi kouqiang yixue zazhi = West China journal of stomatology 2012, 30(1):82-86.

22. Shen $Z$ and Stanger BZ. YAP regulates S-phase entry in endothelial cells. PLoS One. 2015; 10: e0117522.

23. Zhou $\mathrm{X}$, Xing $\mathrm{X}$, Zhang $\mathrm{S}$, et al. Glucose-regulated protein 78 contributes to the proliferation and tumorigenesis of human colorectal carcinoma via AKT and ERK pathways. Oncol Rep. 2016; 36.

24. $\mathrm{Lv} \mathrm{T}, \mathrm{Wu} \mathrm{Y,} \mathrm{Mu} \mathrm{C}$, et al. Insulin-like growth factor 1 promotes the proliferation and committed differentiation of human dental pulp stem cells through MAPK pathways. Arch Oral Biol. 2016; 72: 116-123.

25. $\mathrm{Yu}$ Y, Mu J, Fan $\mathrm{Z}$, et al. Insulin-like growth factor 1 enhances the proliferation and osteogenic differentiation of human periodontal ligament stem cells via ERK and JNK MAPK pathways. Histochem Cell Biol. 2012; 137: 513-25.

26. Lim W, Jeong M, Bazer FW, et al. Curcumin Suppresses Proliferation and Migration and Induces Apoptosis on Human Placental Choriocarcinoma Cells via ERK1/2 and SAPK/JNK MAPK Signaling Pathways. Biol Reprod. 2016; 95.

27. Ishii $\mathrm{T}$, Kohashi $\mathrm{K}$, Iura $\mathrm{K}$, et al. Activation of the Akt-mTOR and MAPK pathways in dedifferentiated liposarcomas. Tumour Biol. 2016; 37: 4767-76.

28. Fausti F, Di Agostino S, Cioce M, Bielli P, Sette C, Pandolfi PP, Oren M, Sudol $M$, Strano S, Blandino G: ATM kinase enables the functional axis of YAP, PML and p53 to ameliorate loss of Werner protein-mediated oncogenic senescence. Cell Death Differ 2013, 20(11):1498-1509.

29. Lee DH, Park JO, Kim TS, Kim SK, Kim TH, Kim MC, Park GS, Kim JH, Kuninaka S, Olson EN et al: LATS-YAP/TAZ controls lineage specification by regulating TGFbeta signaling and Hnf4alpha expression during liver development. Nat Commun 2016, 7:11961.

30. Xie Q, Chen J, Feng H, Peng S, Adams U, Bai Y, Huang L, Li J, Huang J, Meng $S$ et al: YAP/TEAD-mediated transcription controls cellular senescence. Cancer Res 2013, 73(12):3615-3624.

31. Johnson R, Halder G: The two faces of Hippo: targeting the Hippo pathway for regenerative medicine and cancer treatment. Nature reviews Drug discovery 2014, 13(1):63-79.

32. Lian I, Kim J, Okazawa H, et al. The role of YAP transcription coactivator in regulating stem cell self-renewal and differentiation. Genes Dev. 2010; 24: 1106-18.

33. Alarcon C, Zaromytidou AI, Xi Q, Gao S, Yu J, Fujisawa S, Barlas A, Miller AN, Manova-Todorova $K$, Macias MJ et al: Nuclear CDKs drive Smad transcriptional activation and turnover in BMP and TGF-beta pathways. Cell 2009, 139(4):757-769.

34. Varelas X, Sakuma R, Samavarchi-Tehrani P, Peerani R, Rao BM, Dembowy J, Yaffe MB, Zandstra PW, Wrana JL: TAZ controls Smad nucleocytoplasmic shuttling and regulates human embryonic stem-cell self-renewal. Nat Cell Biol 2008, 10(7):837-848.

35. Shimono $M$, Ishikawa $T$, Ishikawa $H$, Matsuzaki $H$, Hashimoto $S$, Muramatsu T, Shima K, Matsuzaka K, Inoue T: Regulatory mechanisms of periodontal regeneration. Microscopy research and technique 2003, 60(5):491-502. 